Fecha de recepción: abril 2021

Fecha de aprobación: mayo 2021 Fecha publicación: junio 2021

\section{Estrategias de inclusión a través del arte y la artesanía en colectivos de mujeres. Una visión crítica}

María Jesús Cano Martínez ${ }^{(1)}$ y

César González-Martín ${ }^{(2)}$

\begin{abstract}
Resumen: Las artesanías presentan un espacio de trabajo idóneo para el desarrollo de proyectos inclusivos dirigidos a colectivos de mujeres vulnerables o en riesgo de exclusión social, donde se posibilita el trabajo comunitario, el desarrollo de capacidades, el empoderamiento y sororidad, así como su desarrollo profesional.

En este estudio veremos las disciplinas artesanales vinculados colectivos de mujeres, observando una mayoría de propuestas orientadas a oficios históricamente feminizados, lo que produce una paradoja en la que por un lado se facilita la integración de la mujer, mientras se continúan instalando estereotipos de género que favorecen la segregación horizontal.
\end{abstract}

Palabras clave: artesanía - inclusión social - mujeres - género - estereotipos.

[Resúmenes en inglés y portugués en la página 132]

(1) María Jesús Cano Martínez es Doctora en Historia y Arte, por la Universidad de Granada. Licenciada en Bellas Artes por la Universidad de Granada, Diplomada en Enfermería por la Universidad de Jaén y Experta en Metodologías Innovadoras en Educación por la Universidad de la Rioja, ejerce como PDI en el Departamento de Enfermería de la Universidad Jaume I (España). Así mismo ha desarrollado otro tipo de docencia en el campo del Arte a través de conferencias, simposios, talleres y publicaciones en medios científicos. canom@uji.es

(2) César González-Martín es profesor e investigador asociado del Departamento de Historia y Ciencias Sociales de la Facultad de Artes Liberales de la Universidad Adolfo Ibáñez (Chile). Doctor en Historia y Artes, Licenciado en Bellas Artes y posgraduado en Producción e Investigación en Artes por la Universidad de Granada (España). Ha participado en proyectos de investigación en el ámbito europeos, nacional y autonómicos. Ha realizado estancias de investigación en Milán (Italia), en la Universidad del Valle de Cali (Colombia) y en la Facultad de Bellas Artes de Tetuán (Marruecos). Es autor de numerosas publicaciones. cesar.gonzalez@uai.cl 


\section{Inclusión e integración. Hacia dónde nos dirigimos}

"Diversidad es que te inviten a una fiesta. Inclusión es que te saquen a bailar". Fundación Adecco $^{1}$

Es más. Hablar de plena inclusión, es que no sientas ser el patito feo mientras bailas. Que sientas incluso que eres la étoile del baile. Al hablar de conceptos como integración e inclusión, encontramos en el primero la intencionalidad de que la persona sea integrada dentro de una comunidad, de modo que ésta se adapte a la misma. Mientras tanto, el segundo, incide en la idea de que, al integrar a la persona en la comunidad, ésta debe ser incluida en toda su individualidad, aceptando su identidad, diversidad y comprendiéndola. El segundo enfoque, que es en el cual se viene trabajando desde la pedagogía (Cardona, 2006; Jiménez, 2008; Booth y Ainscow, 2015), pero también desde otros ámbitos (trabajo social, sanidad, recursos humanos, etc.), donde la diversidad en todas sus vertientes ha de ser contemplada (Aguilar, Gaviria y Laparra, 1995; Peña, 2005; Echeita, 2006; Díez, 2007; UNESCO, 2009; Díez y Pedreño, 2014), parece la opción a seguir. Sin embargo, hemos de ser más ambiciosos y pensar que, si bien hemos de aceptar y comprender la diversidad, debemos trabajar también para una integración e inclusión plena y real de las personas/ colectivos en riesgo de exclusión o vulnerables en las comunidades de destino, abandonando posturas paternalistas y condescendientes que puedan favorecer una perdida de implicación, emprendimiento y liderazgo en estas personas.

Sea cual sea el ámbito de actuación, y teniendo en cuenta las particularidades del colectivo con el cual se trabajarán, las acciones para la inclusión se pueden orientar a su vez hacia diferentes áreas del individuo (Gráfico 1): desde la unidad familiar (ámbito al que se atiende en inclusión desde el ámbito educativo, pero que se ha de tener en cuenta dado que es la principal unidad en base a la cual se construyen las comunidades), al ámbito educativo, social (con toda su complejidad, atendiendo a la configuración de los propios sistemas desarrollados y su acceso, a las relaciones interpersonales o a la propia cultura, tanto de la comunidad receptora, como externa) y laboral.

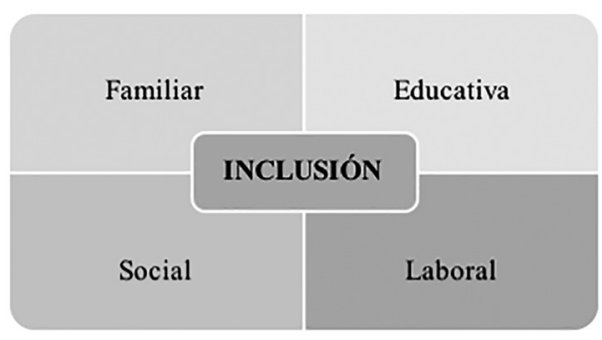

Gráfico 1. Elaboración propia. 
Así mismo, no solamente hemos de tener en cuenta estas áreas de desarrollo personal esenciales para una plena inclusión, sino aspectos como el contexto sociocultural, donde se suceden una serie de pautas, representaciones y/o convenciones sociales, en base a las cuales habitualmente se mide (de forma errónea) el éxito de la inclusión.

En su connotación más usual, la identidad social se concibe como la vertiente subjetiva de la integración. Es la manera como el actor interioriza los roles y estatus que le son impuestos o que ha adquirido y a los cuales somete su "personalidad social". La identidad social es entonces más fuerte si el actor ha integrado bien los sistemas normativos y las expectativas que le son atribuidas por los demás y por el “sistema” (Dubet \& Zapata, 1989, p. 520).

Esto nos llevaría a pensar, que, si planteamos un proyecto de inclusión social para un colectivo determinado, el éxito podría esta vinculado a que el rol desarrollado en el mismo, sea los más afín a los individuos participantes. Sin embargo, la plena inclusión incide en la aceptación de la diversidad, y en tanto la pluralidad de identidades. Nos encontramos entonces, frente a un doble desafío: favorecer la inclusión y el desarrollo de nuevos roles sociales.

Es importante tener en cuenta las diferentes dimensiones que contemplan el proceso de inclusión de un individuo, no sólo en cuanto a áreas de actuación, sino también como aspectos que influyen directamente en el que el proceso se realice de una forma adecuada e igualitaria. Por ello, incluimos (valga la redundancia) el concepto de interseccionalidad, que comenzó a instaurarse a través de los estudios de género y de la importancia de cuestionar los aspectos no solo de género, sino también raciales, y como su convergencia influye en el desarrollo de las mujeres, y en última instancia, del sujeto que se ve condicionado por los aspectos sociales que le rodea. Así, como dice Crenshaw (1991), "hablamos de la necesidad de tener en cuenta los múltiples fundamentos de la identidad al considerar cómo se construye el mundo social”2 (p. 1245).

Así pues, atendiendo a las diferentes dimensiones que contemplan la inclusión, queremos centrar nuestra atención en el ámbito laboral. Y a su vez, no solamente centrarnos en la necesidad de inclusión a nivel laboral, sino de que forma se lleva a cabo esta inclusión, valorando su viabilidad y si se ejecuta de una forma completamente igualitaria ${ }^{1}$.

Y es que, cuando exponemos los aspectos relativos a la inserción e inclusión laboral de la mujer, hemos de tener en cuenta las barreras que aún a día de hoy, se presentan en el ámbito laboral. Principalmente encontramos dos tipos principales de exclusión o de trato desigual con respecto a la mujer: la segregación vertical y la segregación horizontal. La primera hace referencia a las desigualdades o impedimentos a los que las mujeres se enfrentan para acceder a ciertos puestos de trabajo, desafiando las jerarquías establecidas a nivel laboral y remuneración igualitaria co respecto al hombre en puestos con la misma responsabilidad. Por otro lado, la segregación horizontal hace referencia a desigualdades con respecto a los espacios que hombres y mujeres ocupan (Bradley, 1989; Williams, 1986; Reskin \& Hartmann, 1986; Valian, 1998). Así, la división del trabajo, como masculino y femenino se ha presentado de forma continua desde la incorporación de la mujer al ámbito laboral (Benería, 1979; Becker; 1985; Hakim, 1992; Anker, 1998; Kandel, 2006; Cabrera, 2013; Mazzei, 2013). 
Se evidencia de esta manera, que el género incide como factor añadido, al acceso de la mujer a unos ámbitos de trabajo u otros, debido a una construcción paulatina del espacio laboral, donde se han desarrollado estructuras binarias basadas en estereotipos de género. Tal división, se ha visto reflejada en el sector artesanal, estableciéndose divisiones que circunscribían a la mujer en el ámbito de lo doméstico (y en tanto de los oficios relacionados con ese ámbito privado como el textil y desarrollo de objetos asociados al contexto del hogar principalmente) y al hombre, a ámbitos donde primaba la fuerza (trabajo en madera, metalurgia, etc.). Hill citado en Sánchez (2008), hace una revisión sobre la evolución del trabajo de la mujer en el contexto del desarrollo industrial, dónde además se asocia dicha división o segregación horizontal, a la segregación vertical (los "oficios femeninos" presentaban de partida peores condiciones que los "oficios masculinos"): “(...) los llamados oficios de mujer, que incluían a sombrereras, costureras, y modistas, y el "trabajo femenino" se solía remunerar como tal, es decir, resultaba más barato” (p. 75).

\section{Heterogeneidad de problemáticas}

No solo hemos de plantearnos la individualidad de cada mujer, sino a su vez, los diferentes contextos geográficos y socioculturales, que marcan igualmente los proyectos personales de las mujeres y que las ligan a una serie de expectativas. Por ello, no podemos equiparar el enfoque de trabajo con un colectivo de mujeres inmigrantes (con su cultura, sus proyectos migratorios y su historia personal) a un colectivo de mujeres que han padecido violencia de género (en un contexto cultural distinto, con unas redes de apoyo particulares, etc.). Sin embargo, si bien el enfoque y las consideraciones se deben adaptar al grupo de trabajo, la herramienta puede ser considerada como un elemento a parte a explorar. Es por ello que nos cuestionamos hacia qué vías se dirigen los proyectos, que como posteriormente expondremos, se concentran en actividades de autoexploración a través de dinámicas artísticas (fotografía, dibujo, etc.), y en lo artesanal trabajos que se han ligado a lo femenino (textil, cerámica de objetos cotidianos, etc.). Teniendo en cuenta, no solo los beneficios personales, sino la proyección en el futuro, así como aspectos que en la actualidad han de ser necesariamente revisados como la perspectiva de género y/o la sostenibilidad. Todos estos aspectos transversales, se deben integrar a su vez con las particularidades del colectivo con el cual se desarrollará el trabajo.

En este sentido, cabe remarcar el enfoque propuesto por Carrascosa (2010), en el abordaje del trabajo de inclusión con colectivos de mujeres inmigrantes, señalando lo siguiente:

Nuestra propuesta de creación artística como herramienta efectiva de integración social trabaja la integración desde el enfoque interaccionista. Se valora la integración desde la perspectiva de "capacitación hacia el futuro". (...) No consideramos que se trate de armonizar las conductas conforme a los esquemas del país de acogida, sino de desarrollar la capacidad para hacerse valer. El refuerzo de una misma potencia la capacitación para el buen manejo de experiencias (p. 196). 
Cabe pensar, además de los aspectos anteriormente expuestos, si el área de trabajo en el que en ocasiones se desarrollan proyectos de inclusión es el más oportuno (en los términos expuestos: proyección de futuro, perspectiva de género y/o sostenibilidad), lejos de ser el más relacionado históricamente a la mujer, dado que como veremos a continuación, la tendencia es a implantar o trabajar con colectivos de mujeres artesanas, donde la herramienta metodología se asocia a un oficio históricamente "feminizado".

¿Qué capacidad de desarrollo tendrá el aprendizaje del tejido textil?, ¿es un sector en auge en la zona en la que estamos trabajando?, ¿cuáles son los sectores en auge? Estas deberían ser algunas de las preguntas para poder dirigir los esfuerzos hacia un aprendizaje realmente emancipador para estos colectivos y con una visión holística de todos los factores implicados en el desarrollo de un proyecto.

\section{Reivindicación y empoderamiento desde el arte y la artesanía en colectivos de mujeres}

De forma paralela a la idea de utilizar el arte (como práctica per se o como acción de Arteterapia) o la artesanía como medio de inclusión, se viene trabajando desde hace tiempo en las conexiones que tales prácticas se establecen con el desarrollo y empoderamiento de la mujer, además de la propia conexión histórica de lo artesanal (en cuanto a su vinculación con lo doméstico) y la propia figura de la mujer (Luckman, 2013b; Groot, 2015; Leone, 2021). Por ello, es interesante abordar estas prácticas desde una perspectiva de género que sea capaz de comprender, cómo estos vínculos históricos pueden condicionar el desarrollo de diversos proyectos, tanto desde un punto de vista del empoderamiento de la mujer como de la preservación de ciertos estereotipos.

Históricamente, la artesanía principalmente ha sido asociada a la mujer por su vínculo con lo doméstico, siendo el desarrollo de la artesanía textil y cerámica (relacionada con el desarrollo de utensilios domésticos), los oficios íntimamente asociados. Así mismo, con el transcurso del tiempo, y el cambio de modelo social que se produce con la revolución industrial, esta asociación se refuerza, buscando incluso, círculos de actuación al margen de lo exclusivamente privado. Así,

Como forma de influencia social, la artesanía ayudó a las mujeres a tener una mayor presencia dentro de su religión y les proporcionó un medio para apoyar causas benéficas. Las mujeres también aumentaron su influencia social a través de la creación de artesanías basadas en la naturaleza, lo que les permitió ganar un punto de apoyo en el mundo de la ciencia, ya que recogieron especímenes para su trabajo (Bornhorst-Winslow, 2012, p. 1$)^{4}$.

En este contexto, y de forma progresiva, las mujeres toman posición activa de su propia capacidad de acción y organización, de forma que se produce un cambio en el enfoque con respecto a la idea de poder. Así pues, 
El poder es definido como, “(...) poder para", "poder con”, "poder interior”, se está refiriendo a otro entendimiento de poder, como distinto al "poder sobre" en donde el empoderamiento significa la inclusión de la gente en el proceso de toma de decisiones, proceso del que estaba excluida (Corona, 2000; p. 103).

"La identidad de género parece estar estrechamente ligada a la identidad de artesano, y puede considerarse que desempeña un papel diferente según se trate de un artesano o una artesana" (Gauer, 2011, p. 38)

Este vínculo en parte, favorece el trabajo con colectivos de mujeres a través de la artesanía y el arte, lo manual, ya que se establecen relaciones de cercanía, y espacios de desarrollo donde la mujer puede expresarse a la vez que desarrolla una acción (que puede generarse como actividad de refuerzo de los vínculos comunitarios y desarrollo personal, o bien como aprendizaje que favorezca el desarrollo de habilidades y herramientas para una posterior inclusión laboral) (Erazo y Cevallos Moreno, 2019).

\section{Proyectos con colectivos de mujeres: con quiénes nos encontramos y hacia dónde se dirigen}

$\mathrm{Al}$ realizar una búsqueda sistemática de publicaciones científicas que recojan el desarrollo de proyectos vinculados a colectivos de mujeres vulnerables o en riesgo de exclusión, encontramos que existe poca documentación al respecto. Si bien, explorando en fuentes secundarias como sitios web de instituciones vinculadas al desarrollo de la mujer (mujeres migrantes, mujeres indígenas, mujeres víctimas de violencia de género, etc.) figuran diversas experiencias conectadas con el ámbito artístico y artesanal, no se realiza un seguimiento que aporte cuales fueron los objetivos principales, y especialmente, los inputs posteriores a la acción.

Revisando la documentación científica al respecto, encontramos una división principal: proyectos dirigidos a acciones artísticas, que habitualmente se vinculan con objetivos de inclusión a nivel comunitario, autoexpresión y desarrollo personal y proyectos vinculados con la labor artesanal, donde se favorece el empoderamiento de los colectivos de mujeres a través del desarrollo de artesanías vinculadas con su ámbito geográfico y cultural, que tienen como objetivo principal afianzar modelos de negocio sostenible para estas mujeres. Dentro del primer grupo, relacionado con el desarrollo de actividades artísticas y/o Arteterapia, encontramos un incipiente desarrollo de estas acciones asociado a colectivos de mujeres migrantes (Carrascosa, 2010; Rico, 2012; Sánchez, 2012). En su mayoría, estos proyectos posibilitan la expresión de emociones y situaciones concretas y complejas en las cuales estas mujeres se ven inmersas a través del dibujo como principal herramienta artística de expresión. Posiblemente por la economía de recursos y el vínculo con el dibujo que todas las personas hemos tenido en nuestra historia de vida y que con el tiempo hemos perdido. Así mismo, también se han desarrollado este tipo de acciones en colectivos de mujeres en prisión y también colectivos de mujeres con algún problema o patología que las sitúa en una posición de vulnerabilidad social (por ejemplo, el proyecto realizado con 
mujeres alcohólicas rehabilitadas presentado en el trabajo de Omenat (2006). Este tipo de acciones se han venido realizando en el contexto español, donde aun el desarrollo de la Arteterapia no está bien afianzado. Asociado a otra práctica artística, en el límite con el trabajo artesanal, encontramos el trabajo desarrollado por Polanen (2020), en torno a los beneficios del arte textil a nivel emocional.

Con respecto al segundo grupo detectado en la revisión, donde se presentan proyectos relacionados con la inclusión laboral de colectivos de mujeres a través de la artesanía (Corona, 2000; Forstner, 2013; Hemingway, 2013; Jacobs, 1998; Kelly, 2014; Lysen, Langley, Long y Belliard Urena, 2013; Mamani Mendoza \& Ramos Sucapuca, 2018; Márquez Benavides, Latinlán, ; Malema y Naidoo, 2017; Malema y Naidoo, 2017a; Pentney, 2008; Stall \& Stoecker, 1998; Stein y Lourensen, 2018; Tubay, 2019), podemos observar que una gran presencia de proyectos en el contexto de América Latina.

Independientemente de la región geográfica, cabe destacar el tipo de artesanías desarrolladas, en su mayoría artesanía textil, desarrollo de cerámicas y un caso descrito de trabajo con jabón artesanal en el contexto de la Prisión estatal Madre Pelletier en Portugal (Stein y Lourensen, 2018). Este proyecto se centra más en la inserción de los docentes en el programa que en el seguimiento de las actividades de las mujeres, así como en hacer alusión a las conexiones del proyecto con base al ecofeminismo.

Estos proyectos refuerzan el empoderamiento de los colectivos de mujeres, asociados a su contexto geográfico y reivindicando su propia identidad cultural (específicamente en los casos de mujeres indígenas y/o contextos rurales alejados de los grandes núcleos urbanos). Así mismo se favorece el apoyo a dichas comunidades, implementando herramientas que favorezcan el desarrollo laboral, a través de programas de formación.

Destaca de forma evidente, las asociaciones que se establecen a su vez entre trabajo artesanal, empoderamiento y ecofeminismo en el trabajo con estos colectivos, asociación tan potente como en ocasiones, favorecedora del estancamiento en ciertos roles y/o estereotipos. Sin embargo, lo más destacable es como estos programas posibilitan el desarrollo de estas mujeres:

"Tras la formación impartida a estas mujeres, ahora están preparadas para crear sus propias pequeñas empresas y ganarse la vida" (The Express Tribune, 2010) ${ }^{6}$.

\section{Resultados de las experiencias en los diferentes colectivos}

Con respecto a los resultados que arrojan los diferentes estudios en revisión, podemos apuntar a diferentes líneas de desarrollo para la mujer, todas ellas fundamentales para favorecer la inclusión a todos los niveles descritos en nuestra introducción. Es importante recordar el carácter holístico del proceso de inclusión, así como el fenómeno de interseccionalidad que se produce en los colectivos de mujeres para entender que todos los efectos que tales proyectos generan, mejoraran los procesos de inclusión, aunque no siempre se dirijan a aspectos concretos como lo sería la inclusión laboral per se.

Con respecto a los proyectos que emplearon la práctica artística como vehículo de inclusión, encontramos una serie de beneficios que en primera instancia surgen del propio tra- 
bajo grupal, y el contacto con otras mujeres en las mismas circunstancias (favoreciéndose de partida la sororidad en estos colectivos). Omenat (2006), refiere como “(...) a través del proceso grupal, las mujeres han podido descubrir que a la par que evolucionan sus dibujos, pueden introducir pequeños cambios para mejorar sus vidas" (p. 147). Los procesos artísticos en sí mismos posibilitan, como hemos apuntado anteriormente, la expresión de emociones y vivencias desde un lenguaje distinto, pero a su vez, emancipador. Esto contribuye al desarrollo de los colectivos de mujeres que han sufrido vivencias complejas o de exclusión, y si bien, en ocasiones el objetivo principal es el de apoyo, la propia evolución de la mujer consigo misma y sus circunstancias, posibilita en ella una acción de cambio. Así mismo, los proyectos realizados por Carrascosa (2010), Rico (2012) y Sánchez (2012) con mujeres migrantes, los cuales se basaron en acciones arteterapéuticas a través del dibujo, las autoras señalan efectos similares: libertad de expresión de las emociones a través del dibujo, cambios en su propio proceso y auto-reconocimiento de la propia identidad, autoconocimiento, refuerzo con el trabajo grupal.

En relación a los proyectos enfocados exclusivamente al trabajo artesanal el núcleo central de actuación es promover el aprendizaje y emprendimiento de los colectivos de mujeres. Así por ejemplo, dentro del proyecto realizado en la comunidad de Pang Ung Mhai, en Bangkok, con mujeres artesanas de la zona (Lysen, Langley, Long y Belliard Urena, 2013), éstas señalaban un alto grado de satisfacción con las competencias adquiridas en campos relativos al marketing y herramientas de emprendimiento.

Sin embargo, otra serie de beneficios surgen a raíz del trabajo con estas mujeres. En este sentido, como eje principal de estos proyectos, encontramos el propio empoderamiento que experimentan estas mujeres; esa sensación de apoyo mutuo y de capacidad personal que les posibilita desarrollar las acciones con pleno convencimiento. Malema y Naidoo (2017) concluyen que los proyectos comunitarios de arte y artesanía contribuyen positivamente al empoderamiento de las mujeres, pero requieren un mayor apoyo de las estructuras disponibles para garantizar los beneficios sostenidos de la participación de las mujeres rurales. Es importante como se apunta a un mayor compromiso en el mantenimiento $a$ posteriori de los proyectos.

Observamos como algunos proyectos tienden a incorporar el ecofeminismo en su desarrollo teórico y en la reafirmación de la figura de la mujer (Valle, 2019). Sin embargo, tal línea puede caer en la prolongación de estereotipos, ya que se sigue ligando a la mujer con lo doméstico, como extensión del trabajo de la tierra. Esta idea queda reforzada por autores como Pentney (2008), que realiza un estudio sobre las asociaciones que se establecen entre activismos y movimientos feministas y resalta "la reclamación y celebración de la artesanía feminizada". O bien Tubay (2019):

Tanto el artesanato como el arte parecen haber adquirido algunas etiquetas que fomentan la desigualdad, vinculando cuestiones de raza, origen, sexo y clase social a su actividad, propiciando cualidades hegemónicas a unos y restándoselas a otros. (...) ahora hay más chicas que empiezan a gustar de la costura, normalmente eran las personas más viejas que tenían ese hábito. Pero ahora cada vez más mujeres quieren aprender a coser, a tener una marca propia. Creo que las cosas ahora son mejores (p. 11). 


\section{¿Estamos cerca de una plena inclusión o seguimos perpetuando patrones de segregación horizontal?}

Habiendo observado la evidente "feminización" de los proyectos desarrollados en colectivos de mujeres, no tanto por su propia constitución, sino por el oficio elegido a desarrollar, llegamos a discutir nuestra hipótesis de estudio principal, que es realizar un análisis crítico de por qué se perpetúan ciertos estereotipos y apreciando como se plantea necesario revisar las connotaciones de género en relación con los proyectos desarrollados con colectivos de mujeres.

Cuando se presentan casos donde la propia tradición es la que lleva a la mujer a seguir desarrollando el trabajo aprendido en el ámbito doméstico (artesanía desarrollada en el seno familiar o actividades manuales aprendidas de la figura materna), podemos encontrar sentido a la prolongación de estereotipos asociados al género vinculados a la artesanía. Pero, ¿por qué cuando se diseña un nuevo proyecto, donde no existen referentes de partida, sino una serie de recursos disponibles, volvemos a caer en la estereotipación?

En general, ninguno de los proyectos evaluados hace una revisión de la propia perspectiva de género en el desarrollo de las prácticas, aun trabajando con colectivos de mujeres. Una visión más global e integradora, no solamente podrá favorecer una real inserción de los diferentes colectivos, sino un aporte social, capaz de reconfigurar los perfiles asociados a las disciplinas artesanales, como parte del engranaje social.

Con respecto al trabajo desarrollado en diversas comunidades en el contexto de América Latina, se extraen las siguientes metodologías e intervenciones (con una visión de género en su desarrollo):

a. el cuestionamiento crítico de la condición subordinada y discriminada de las mujeres;

b. la estimulación de la capacidad crítica y de reflexión con el fin de encontrar propuestas transformadoras;

c. la toma de conciencia y el reforzamiento de la identidad de género de las mujeres como un proceso de aprendizaje colectivo;

d. encontrar una identidad colectiva que refuerza la participación de las mujeres;

e. tener a su realidad y experiencias como punto de partida, $y$

f. relacionar género con clase y etnia, ubicar la identidad de las mujeres en un contexto más amplio, en varias esferas de la vida y de la sociedad (Corona, 2000; p. 114).

En este sentido, cabría cuestionarse, en qué sentido se trabaja para reforzar la identidad de género, ya que está en sí, es una entidad subjetiva e individual. El hecho de centrarse solamente en una identidad, como una identidad global de lo que significa ser mujer, en cierto modo cae en una privación de la diversidad de identidades, así como la inclusión de las mismas, y en el posible riesgo de afianzar ciertos estereotipos o roles asociados a la figura de la mujer.

En los primeros movimientos del feminismo, algunas voces presentaron su desacuerdo con respecto a la artesanía asociada a lo doméstico. Mary Wollstonecraft (1995) o Hannah More (1856) ya establecía una asociación de opresión con la artesanía doméstica, por la continuación del reducto mujer-entorno doméstico. 
Ciertamente, el vínculo de la mujer con el trabajo artesanal le ha brindado nuevas posibilidades de expresión, relación, conexión con la propia comunidad y divulgación de su propio trabajo. Obviamente los beneficios están ahí. Pero quizás la pregunta sería: ¿no disfrutarían de los mismos beneficios siendo partícipes de otras disciplinas? y ¿por qué seguimos instalados en estereotipos dicotómicos asociados al trabajo?

Como bien señala Fornster (2012), en relación al trabajo desarrollado en ámbitos rurales, "a pesar de que tanto hombres como mujeres pueden ser artesanos, la artesanía dentro del ámbito rural suele ser presentada como una actividad económica que es predominantemente femenina" (p. 143), enfatizando como razón que favorece esta realidad el hecho de “(...) que la artesanía es una actividad flexible que pueden combinar con otros trabajos, productivos y reproductivos" (Ibid; p. 150). En este sentido, también Leone (2021) establece dicha asociación entre artesanía y ámbito rural haciéndonos cuestionar la propia clasificación de los colectivos, tendiéndose a relegar la artesanía junto con lo rural (no urbano, menos sofisticado, menor nivel) y lo femenino.

Obviamente no se trata de apartar a la mujer de oficios feminizados. La idea es poder incluirla en aquellos asociados a un perfil masculino, de forma que se vaya eliminando progresivamente la segregación horizontal, y por otro lado, hacer que los oficios feminizados, en la línea de los proyectos que están trabajando en el empoderamiento de la mujer a través de los mismos, pierdan la connotación de inferioridad que históricamente se les ha podido añadir, ya que no podemos perder de vista esta realidad. Citando a Nelson y colaboradores, (Gauer, 2011), “(...) la estrecha relación entre la artesanía y el género puede significar que la artesanía como "trabajo de mujeres" sigue estando devaluada hoy en día” (p. 32)

\section{Conclusiones}

En base a la revisión de artículos que versen sobre el empleo del arte y la artesanía, como vehículo de inclusión, concretamente en colectivos de mujeres en riesgo de inclusión social, podemos resaltar principalmente la necesidad de hacernos eco de los ya presentes, de una forma sistemática y rigurosa.

Desde asociaciones de mujeres y organizaciones (tanto a nivel institucional como de carácter no lucrativo), se vienen estableciendo diferentes propuestas de inclusión, que pasan por el desarrollo de actividades ligadas al arte, la Arteterapia y la artesanía. Sin embargo, estos no se documentan de una forma científica, ni se valoran los resultados a largo plazo. Así, se hace necesario que tales proyectos, se acompañen de un seguimiento académico que refuercen las dinámicas propuestas y evidencien los múltiples beneficios para estos colectivos.

Con respecto a los estudios incluidos en nuestra investigación, son diversas las conclusiones que obtenemos, de forma colateral a la evidente feminización de las propuestas (aspecto que desarrollaremos de una forma más detenida al final de estas conclusiones). Así pues, encontramos una falta de proyectos concretos y documentados a nivel científico, que asocien estrictamente artesanía e inclusión. Ciertamente, hemos observado una gran presencia de proyectos que trabajan con colectivos de mujeres desarrollando la artesanía 
como medio empoderador. Sin embargo, la artesanía es algo inherente a estos colectivos, en función de su ubicación geográfica y sus aspectos culturales. Es por ello, que sería interesante fomentar aun más la artesanía, como herramienta de inclusión (lo cual se está haciendo con otros colectivos generando experiencias de éxito) en los colectivos de mujeres, otorgándoles herramientas para su propio emprendimiento, así como el aprendizaje de un oficio, que como hemos visto, además genera una serie de beneficios más allá de los meramente laborales.

Con respecto a las iniciativas desarrolladas desde el Arteterapia, igualmente observamos como existen aún muchos campos donde desarrollar el trabajo, que favorezca el desarrollo de las mujeres vulnerables, en áreas artísticas que no solamente se circunscriban al dibujo. En cuanto al tipo de metodologías que se proponen, las actividades que se plantean como herramienta de inclusión para las mujeres se orientan básicamente al aspecto emocional, con el fin de aumentar su empoderamiento y autopercepción, pero sin plantear proyectos de largo recorrido donde se pueda valorar una inclusión a largo plazo. Esto en sí, es un elemento positivo de las propuestas, pero la relación de los colectivos de mujeres en riesgo de exclusión con el arte, pueden ir más allá, entendiendo el arte, no solo como medio de expresión, sino como otra posible actividad laboral, por lo que no habríamos de desdeñar dicho aspecto.

Ahora bien, centrándonos en el enfoque desde el cual se plantean los proyectos, observamos la paradoja, de que se bien están atravesados por la cuestión del género, en tanto que trabajan por la inclusión de la mujer, no tienen en cuenta aspectos propios de los estudios de género como hemos observado. Así, la mayoría de los proyectos presentan como disciplina central, aquellas históricamente denominadas como feminizadas o asociadas a la figura de la mujer.

A pesar de ser pocos los proyectos difundidos en la comunidad académica, se evidencia que la presencia femenina está presente en proyectos de Arteterapia (donde el dibujo es la herramienta principal) y proyectos relacionados con el ámbito textil, cerámica (asociada a uso cotidiano, con su correspondiente vínculo con lo doméstico) y algunos proyectos aislados como la producción de jabones (nuevamente encontramos ese vínculo con lo cotidiano, lo doméstico y la producción de elementos que se asocian a prácticas habitualmente entendidas como femeninas). Se evidencia así, una prolongación de la estereotipación de los oficios artesanales, la segregación horizontal, y una ausencia de la creación de referentes en otras áreas, especialmente en la artesanía, donde más se presenta este carácter binario con respecto a ciertos oficios. Habríamos de cuestionarnos sí esta elección se justifica por ser sectores donde la demanda es superior y ello pueda influir en una futura inserción laboral. Sin embargo, hemos de tener en cuenta que esa inclusión laboral, incluso en sectores en expansión como el textil asociado a la moda, presentan un efecto de segregación vertical cuando se trata de llegar a puestos de liderazgo, lo que nos lleva nuevamente a posicionar a la mujer en niveles inferiores y no tan ligados a lo público.

Es importante tener en cuenta como se refleja el hecho de la desigualdad, no solamente entre hombres y mujeres, sino en la misma artesanía, como se apuntan en ciertos estudios. Como hacemos para reforzar doblemente estos proyectos: haciendo de las mujeres independientes y de la artesanía un oficio con proyección. No solo podemos centrarnos en las fases de diseño de los proyectos y en el refuerzo de sus beneficios pedagógicos y emociona- 
les, sino aportar un análisis global, capaz de vincular un proyecto con su propia viabilidad, que finalmente será la que aporte al colectivo de mujeres una proyección de futuro. Y todo ello, por supuesto teniendo en cuenta aspectos que atraviesan a día de hoy todo proyecto. Se hace patente la necesidad de aportar una perspectiva de género en el desarrollo de proyectos en general, y con más ahínco en aquellos desarrollados en colectivos de mujeres. Y a su vez, plantear nuevas direcciones que aporten esa proyección de futura necesaria para la inclusión, de modo que se tengan en cuenta aspectos como la incorporación de herramientas digitales para el aumento de la proyección del trabajo artesanal (Luckman, 2013a) y la posibilidad de trabajar con microempresas dedicadas a la neo-artesanía e implicar otros sectores, como puede ser el arte y el diseño favoreciendo la co-creación y el trabajo colaborativo, de forma que tales proyectos tenga un carácter transdisciplinar capaz de aportar a su vez innovación como elemento de emprendimiento y en tanto, una inclusión consciente y duradera para estos colectivos.

\section{Notas}

1. Esta frase fue pronunciada por Verna Myers consultora de Diversidad y actual VP Inclusion Strategy de Netflix.

2. Traducción propia. Texto original: “(...) the need to account for multiple grounds of identity when considering how the social world is constructed".

3. Hacemos este inciso antes de exponer los trabajos que desde el arte y la artesanía se desarrollan en colectivos de mujeres, para establecer esa valoración crítica que supone nuestro objetivo principal.

4. Traducción propia. Texto original: "As a form of social influence, crafts helped women gain a greater presence within their religion and provided them a means of supporting charitable causes. Women also increased their social influence through the creation of nature-based crafts, which enabled them to gain a foothold in the world of science as they collected specimens for their work".

5. Traducción propia. Texto original: From the analysis of the data, gender identity seemed to be closely knit to a crafter identity, and might be seen to play a different role depending on whether one was a male or female crafter.

6. Traducción propia. Texto original: After giving training to these women they are now poised to set up their own small business enterprises and earn their livelihood.

7. Traducido del original: "The close association between crafts and gender may mean that crafting as "woman's work" continues to be devalued today" (Nelson et al., 2005).

\section{Bibliografía}

Anker, R. (1998). Gender and Jobs: Sex Segregation of Occupations in the World. Geneva: International Labour Office. 
Aguilar, M.; Gaviria, M. y Laparra, M. (1995). Aproximación teórica al concepto de exclusión. En AA.VV. (1995). Desigualdad y pobreza hoy. Madrid: Talasa.

Becker, G. S. (1985). Human capital, effort, and the sexual division of labor. Journal of labor economics, 3 (1, Part 2), S33-S58.

Benería, L. (1979). Reproduction, production and the sexual division of labour. Cambridge Journal of Economics, 3 (3), 203-225.

Bornhorst-Winslow, C. (2012). The Important Role Played by Household Crafts in the Lives of Nineteenth-Century Women in Britain and America.

Booth, T. \& Ainscow, M. (2015). Guía para la Educación Inclusiva: desarrollando el aprendizaje y la participación en los centros escolares, 21-52, Madrid, OEI- UHEM.

Bradley, H. (2013). Gender: Key concepts (2nd Ed.). Polity Press.

Cabrera, D. O. (2013). De la división sexual del trabajo hacia la redefinición de las prácticas de cuidado: una experiencia de Economía Solidaria en Cataluña. Summa psicológica UST, 10 (1), 37-47.

Cardona, M. C. (2006). Diversidad y educación inclusiva: enfoques metodológicos y estrategias para una enseñanza colaborativa. Madrid: Pearson-PrenticeHall.

Carrascosa, M. (2010). Creación artística, inmigración y género. Papeles de artetarpia y educación artísitica para la inclusión social, (5), 185-202

Corona, B. M. (2000). Género, empoderamiento y sustentabilidad: una experiencia de microempresa artesanal de mujeres indigenas (No. 2). Grupo Interdisciplinario sobre Mujer, trabajo y pobreza.

Crenshaw, K. (1991). Mapping the Margins: Intersectionality, Identity Politics, and Violence against Women of Color. Stanford Law Review, 43(6), 1241.

Díez, A. M. (2007). La exclusión social: análisis y propuestas para su prevención. Fundación Alternativas.

Díez, E. R. \& Pedreño, M. H. (2014). Acompañar los procesos de inclusión social. Del análisis de la exclusión a la intervención social. Trabajo social, 16 (16), 143-156.

Dubet, F. \& Zapata, F. (1989). De la sociología de la identidad a la sociología del sujeto. Estudios sociológicos, 7 (21), 519-545.

Echeita, G. (2006). Educación para la inclusión o educación sin exclusiones. Madrid, España: Narcea.

Edwards, C. (2006). 'Home is where the art is': Women, handicrafts and home improvements 1750-1900. Journal of design history, 19 (1), 11-21.

Fernández, M. (1998) A propósito de Mary Wollstonecraft. Cuadernos de Historia Contemporánea, 20, 273-287.

Forstner, K. (2013). La artesanía como estrategia de desarrollo rural: el caso de los grupos de artesanas en la región de Puno (Perú). Cuadernos de Desarrollo Rural, 10 (72).

Fundación Adecco (3 de septiembre de 2020) Diferencias entre Diversidad e Inclusión. Fundación Adecco. Disponible en: https://fundacionadecco.org/azimut/diferencias-entre -diversidad-e-inclusion/

Gauer, M. A. N. (2011). Crafting Identities: Female crafters and their expressions of identity through crafts (Master's thesis). 
Groot, M. (2015). Inscribing women and gender into histories and reception of design, crafts, and decorative arts of small-scale non-European cultures. Journal of Art Historiography, (12), 1.

Hakim, C. (1992). Explaining trends in occupational segregation: the measurement, causes, and consequences of the sexual division of labour. European sociological review, 8(2), 127-152.

Hemingway, P. (2013, November 16). Knit is a feminist issue. The Knitting Genealogist -Hemingway \& Hunt. Disponible en: https://theknittinggenie.com/2013/11/16/knitis-a-feminist-issue/

Jacobs, M. D. (1998). Shaping a New Way: White Women and the Movement to Promote Pueblo Indian Arts and Crafts, 1900-1935. Journal of the Southwest, 187-215.

Jiménez, M. (2008). Aproximación teórica de la exclusión social: complejidad e imprecisión del término. Consecuencias para el ámbito educativo. Estudios pedagógicos (Valdivia), 34(1), 173-186.

Kandel, E. (2006). División sexual del trabajo ayer y hoy. Buenos Aires. Editorial Dunken.

Kelly, M. (2014). Knitting as a feminist project? Women's Studies International Forum, 44, 133-144. https://doi.org/10.1016/j.wsif.2013.10.011

Leone, L. (2021). Craft in Art Therapy. Diverse approaches to the transformative power of craft materials and methods. Routledge.

Lysen, A. T.; Langley, C. E.; Long, M. A., \& Belliard Urena, B. D. (2013). Empowering the Women's Cooperative of Ban Pang Ung Mhai through the Competitive Marketing of Crafts.

Luckman, S. (2013a). The aura of the analogue in a digital age: Women's crafts, creative markets and home-based labour after Etsy. Cultural Studies Review, 19(1), 249-70.

Luckman, S. (2013b). Precarious labour then and now: The British arts and crafts movement and cultural work revisited (Doctoral dissertation, Routledge).

Mamani Mendoza, Y. Y., \& Ramos Sucapuca, D. B. (2018). Generación de empleo mediante la producción artesanal de mujeres de escasos recursos económicos de la cooperativa artesanal Modalpaca Puno 2016.

Márquez Benavides, M. M. (2016). Latinlán: una aproximación ecofeminista a la artesanía Sanjacintera en el postconflicto. El caso de las artesanas de San Jacinto. [Trabajo de Grado] Pontificia Universidad Javeriana de Bogotá

Martínez, C. M., \& García, A. G. (2020). Tecendo identidades. Um diálogo entre arte e artesania. Revista GEARTE, 7(3).

Malema, D. R., \& Naidoo, S. (2017). Spaces for the empowerment of women: Rural Arts and Crafts Projects. African Journal of Hospitality, Tourism and Leisure, 6(2), 1-18.

Malema, D. R., \& Naidoo, S. (2017a). The role of community arts and crafts in the empowerment of women living in a rural environment. World Leisure Journal, 59(sup1), 54-60

Mazzei, C. (2013). Producción y Reproducción: la mujer y la división sociosexual del trabajo./Production and Reproduction: women and socio-sexual division of labor. Revista Rumbos TS. Un espacio crítico para la reflexión en Ciencias Sociales, (8), 128-142.

More, H. (1856). The Complete Works of Hannah More (Vol. 2). JC Derby.

Moss, P., \& Maddrell, A. (2017). Emergent and divergent spaces in the Women's March: the challenges of intersectionality and inclusion. 
Novo-Corti, I.; Varela-Candamio, L., \& García-Álvarez, M. T. (2014). Breaking the walls of social exclusion of women rural by means of ICTs: The case of 'digital divides' in Galician. Computers in Human Behavior, 30, 497-507.

Omenat, M. (2006). Arteterapia: una experiencia de grupos de apoyo a mujeres. Intervención realizada en una Asociación de alcohólicos rehabilitados. Papeles de arteterapia y educación artística para la inclusión social. (1), 137-148.

Pasquel Erazo, V. N., \& Cevallos Moreno, P. M. (2019). El empoderamiento femenino a través de la artesanía (Bachelor's thesis, Quito).

Pentney, B. A. (2008). Feminism, activism, and knitting: Are the fibre arts a viable mode for feminist political action? Thirdspace: A Journal of Feminist Theory \& Culture, 8(1). http://journals.sfu.ca/thirdspace/index.php/journal/article/view/\%20pentney/210

Peña, C. (2005). Igualdad educativa y sociedad democrática. En Políticas educativas y calidad. Reflexiones del Seminario Internacional pp. 21-31l. Santiago de Chile: Fundación Ford, Universidad Alberto Hurtado, UNICEF, UNESCO.

Pöllänen, S. H., \& Weissmann-Hanski, M. K. (2020). Hand-made well-being: Textile crafts as a source of eudaimonic well-being. Journal of Leisure Research, 51(3), 348-365.

Powers, M. G., \& Holmberg, J. J. (1978). Occupational status scores: Changes introduced by the inclusion of women. Demography, 15(2), 183-204.

Reskin, B., \& Hartmann, H. (1986). Women's work, men's work sex segregation on the job. National Academy Press.

Rico, L. (2012). Arte, terapia y mujeres migrantes. Caso Kauthar. Papeles de arteterapia y educación artística para la inclusión social. (7), 141-151.

Stall, S., \& Stoecker, R. (1998). Community organizing or organizing community? Gender and the crafts of empowerment. Gender \& Society, 12(6), 729-756.

Stein, J., \& Lourensen, A. D. R. (2018). Grupos de artesanato com mulheres: sabonetes artesanais e ecofeminismo. Salão de Extensão (19.: 2018: Porto Alegre, RS). Caderno de resumos. Porto Alegre: UFRGS/PROREXT, 2018.

The Express Tribune (23 de diciembre de 2010). Cultural exhibition: Connecting people through crafts 'Connecting People through Crafts' organised at Lok Virsa by UNESCO. Disponible en: https://tribune.com.pk/story/93466/cultural-exhibition-connecting-peo ple-through-crafts

Tubay, F. M. (2019). Gender stereotypes: Perspectives in the craft professions in Portugal. Revista Estudos Feministas, 27(2).

UNESCO (2004) CBR: A Strategy for Rehabilitation, Equalization of Opportunities, Poverty Reduction an Social Inclusion of People with Disabilities (Joint Position Paper). Disponible en: https://apps.who.int/iris/handle/10665/43060

UNESCO. Organización de las Naciones Unidas para la Educación, la Ciencia y la Cultura (2009). Directrices sobre políticas de inclusión en la educación. Recuperado de http:// unesdoc.unesco.org/images/0017/001778/177849s.pdf

UNESCO (2010) Empowering women through crafts. Unesco-norway funded project: mapping of cultural assets in districts multan \& Bahawalpur. Assignemt report. Disponible en: https://cupdf.com/document/empowering-women-through-crafts.html 
Valle, L. D. P. (2019). Reflexões sobre prácticas de artesania ecofeminista e pedagogia ambiental. Por uma política da natureza humana e não-humana. Saberes y prácticas: Revista de Filosofía y Educación, 4, 1-19.

Valian, V. (1998). Why so slow? The advancement of women. London: The MIT Press, Cambridge, Massachusetts.

Williams, C. (1989). Gender differences at work: women and men in nontraditional occupations. University of California Press.

Wollstonecraft, M. (1995). Wollstonecraft: A Vindication of the Rights of Men and A Vindication of the Rights of Woman and Hints. Cambridge University Press.

Abstract: Crafts present an ideal working space for the development of inclusive projects aimed at groups of vulnerable women or those at risk of social exclusion, where community work, capacity building, empowerment and sisterhood, as well as their professional development, are made possible.

In this study we will look at craft disciplines linked to women's collectives, observing a majority of proposals oriented towards historically feminised trades, which produces a paradox as it facilitates the integration of women while continuing to establish gender stereotypes that favour horizontal segregation.

Keywords: craft - social inclusion - women - gender - stereotypes.

Resumo: $\mathrm{O}$ artesanato representa um espaço de trabalho apropriado para o desenvolvimento de projetos inclusivos dirigidos a grupos de mulheres vulneráveis ou em risco de exclusão social, onde o trabalho comunitário, o desenvolvimento de capacidades, o empoderamento e a irmandade são possibilitados, bem como o desenvolvimento profissional. Em nosso estudo veremos as disciplinas artesanais vinculadas a grupos de mulheres, observando uma maior parte de propostas voltadas para os ofícios historicamente feminizados, o que produz um paradoxo em que, por um lado, a integração das mulheres é facilitada, enquanto os estereótipos de gênero que favoreçam a segregação horizontal continuam a ser instalados.

Palavras chave: artesanato - inclusão social - mulheres - gênero - estereótipos.

[Las traducciones de los abstracts fueron supervisadas por el autor de cada artículo] 